\title{
Wide Area Cyclic Blackout Mitigation by Supply- Demand Matching of HVAC Counterpart Loads
}

\author{
Kasim Al-Salim \\ Ivan Andonovic \\ Craig Michie \\ Electronic and Electrical Eng. Dept., University of Strathclyde, Glasgow, G1 1XQ, UK \\ kasim.al-salim@strath.ac.uk i.andonovic@strath.ac.uk c.michie@strath.ac.uk
}

\begin{abstract}
Many countries around the world are challenged to meet the escalating demand for power often resulting in frequent blackouts. Domestic standby generation and associated running costs are prohibitive and novel strategies to provision measures that manage blackouts are becoming much sought after. Almost all installed standby generation is not fully utilized and certain amounts of surplus power can be identified. The paper presents a strategy that harnesses the aggregated standby superfluous power to fulfil essential demand in residential areas during cyclic blackouts covering wide areas. The solution has at its foundation, a multiagent distributed demand management system with a supplydemand matching capability. Environmental conditions are monitored periodically and power is distributed accordingly to each sub-district. Customers at sub-districts receive a share of power according to two different distribution criteria and although their immediate allocated power is not the same, their overall daily power ration is equal. Air conditioners are backed up with less power demanding counterparts and a group of options is adaptively clustered. Their usage rights are distributed among customers according to available superfluous power. The approach is evaluated through an extensive emulation framework and results show that the proposed system is capable of providing an acceptable Quality-of-Service (QoS) level during cyclic blackout periods.
\end{abstract}

Keywords - Blackouts; Blackout Mitigation; Multi-Agent Systems; Demand Side Management; Supply Demand Matching; Power Management, Distributed Generation Aggregation.

\section{INTRODUCTION}

Modern technological breakthroughs have resulted in the extensive use of a spectrum of electrical devices which in turn have created an escalating demand for power. In addition, the repetitive nature of routine daily life features bursts of activities forming demand peaks which in many cases result in exceeding the available power and in turn blackouts. Many countries around the globe have witnessed large scale blackouts, the most striking being in India which affected 700 million users [1]. Deploying additional power generation capability is becoming prohibitively costly with often a negative impact on the environment. One alternative is to redistribute demand evenly throughout the day, in so doing eliminating the need for peak load generation and satisfying the demand with base load only. This motivation has stimulated a plethora of load management techniques, measures and strategies, Demand Side Management (DSM) being the most noteworthy. Demand is modulated using various principles such as peak clipping, valley lifting, load shifting, demand conservation and build-up. Customers are essential participants in all solutions; all successful deployments rely on end user approval and collaboration. Blackouts can be precipitated by a range of events, the most obvious being escalating demand resulting in a 'peak time' blackouts. The severity of the power shortage specifies how deep or long a blackout is. If the power shortage continues for extended periods of time, the blackout migrates into a cyclic mode in which power is provided according to an ON/OFF ratio. Finally, if the power system reaches a steady state at which point generation always lags demand for an extended period of time (years), the cyclic blackout evolves to the most severe chronic mode. This mode is as a result of a wide scale degradation of the installed power generation, transmission and/or distribution sub-systems owing to post-war or post-disaster periods; Iraq is a recent example.

A significant body of research has focused on analyzing, and proposing solution to blackouts and their triggering factors. [2], [3] [4] [5] studied blackouts, their causes, mitigation, and prevention from the power system viewpoint. Others focused on proposing preventive measure such as intentional islanding [6], [7], [8], load shedding [9], [10], and micro grids [11]. All these solutions power a subset of the customers in an attempt to survive a blackout. Sacrificing customers' welfare during short period blackouts is acceptable but severe power shortages causing chronic cyclic blackout are not. On the other hand, [12] tries to reshape demand by resetting air conditioners' thermostats in order to prevent a blackout which is not effective in hot countries. Other such as [13] suggest measures to reduce the demand such as cooling the roofs in order to reduce air conditioning demand. Multi-agent systems are proven to be effective in distributed problems such as power management. [14] propose an agent-based supply-demand matching for renewables interacting with the power market. [15] propose aggregating distributed generators while [16] describe the potential impact of aggregating available standby generation. [17] suggest adding a complete set of distributed generation, energy storage, communication infrastructure, and controllers integrated into a Quality Control Center to improve power quality and provide power during emergencies, an expensive solution if it is used to sustain high loads.

The wide spectrum of available research with all its techniques and strategies can be classified into two approaches; 'blackout prevention' and 'blackout containment'. The first can be defined as a set of DSM measures undertaken to prevent a blackout from occurring based on shedding load either directly or indirectly in order to sustain the remaining load, negotiating solely with the utility. The second can be defined as a set of measures undertaken to contain a blackout from impacting the entire grid through (say) islanding; blackout containment also negotiates with the utility only. Alternatively, blackout mitigation so far is used to describe a set of measures taken to reduce the probability of having a blackout such as permitting a minimum number of transmission lines to overload, increasing transmission line emergency ratings, and increasing generation margins by 
installing more generation [3]. In this paper blackout mitigation is viewed from the customer view point, thus 'blackout mitigation' can be defined as a set of measures undertaken during a blackout to enable users to retain a certain Quality-of-Service (QoS). Here a QoS level is defined as the difference between the current living environment resulting from operating a certain HVAC appliance and the target environment that provides a degree of satisfaction to the customer [18]. Blackout mitigation neither changes nor uses direct utility power and does not correct the blackout. The acquisition of a standby generator is an option but operational challenges such as noise, polluting emissions, fire hazard of stored fuel, frequent costly maintenance, oil disposal problems are factors which prohibit deployments. Renewable options are more attractive in this respect but are tied tightly to weather and although they are environment-friendly they still remain costly, especially if they are used to operate air conditioning systems.

The contribution of the paper, inspired by [14] and [16], is to provide a solution to chronic cyclic blackouts covering wide areas using limited utility and variable superfluous power inherent in existing scattered standby generation capabilities enabling customers to operate appliances including HVAC in environments where air conditioning is a must. The approach presented here relies on the polling of all neighborhood standby generation facilities - regardless of ownership - to map the level of superfluous power. This surplus is then used to power the 'basic set' of appliances such as lights, refrigerators and targets the need to meet 'air conditioning' demand. The basic appliance set does not include high power consumption appliances such as electric cookers and is confined to the provision of one HVAC appliance (either an (AC), an air cooler (CO), or a mist fan (MF)) in order to create an acceptable living environment. COs are evaporative coolers which use water to cool the air, while MFs are fans equipped with water sprayers; a typical $\mathrm{CO}$ consumes around $400 \mathrm{~W}$ and an MF consumes around $150 \mathrm{~W}$. All dwellings are assumed to be equipped with at least one $\mathrm{CO}$ and an MF.

The type of HVAC used depends on the level of available surplus power. Thus the principle is that each dwelling is allocated a level of $\mathrm{AC}, \mathrm{CO}$, and $\mathrm{MF}$ operational time following a fair distribution referred to as the 'usage right' i.e. the right to use a certain HVAC appliance for a specified time. The optimum solution is when there is sufficient power to allocate an AC to every dwelling throughout the day. If this criterion cannot be satisfied the solution finds the optimum blend of ACs, COs, and MFs that matches available surplus. The system ensures that during the entire day, each dwelling has at least one HVAC appliance operational and has used each one of the allocated HVAC options for the same length of time as needed.

Since proper operation depends on the humidity level which obviates the use of COs and MFs and to ensure the best QoS level, humidity is monitored throughout the provisioned area and power is allocated to different sub-districts according to that humidity level at their location. Areas with high humidity such as near the coast during certain summer days should be allocated sufficient power to operate all their ACs on a one per customer basis. This power is provided from the limited utility not from standby surplus power. Furthermore, customers in less humid areas have less utility share but more standby surplus power necessary to operate one of the three HVAC appliances, i.e. humid areas depend entirely on utility supply while the remaining areas depend on a combination of remaining utility and surplus standby power. Adaptive clustering of HVAC appliances with each power level ensures that each customer has the usage rights to operate a single HVAC appliance for a certain period during the day.

The proposed system is backed up by a case study with Iraq chosen for this purpose due to several factors; firstly, it has suffered from chronic cyclic blackouts since 1996 and secondly, it boasts a significant amount of standby generation. In addition, Iraq has a wide diversity of geographies including coastal, desert, plain, swamp, and mountainous areas organised in 19 provinces, 120 districts, and 290 sub-districts. This diversity results in diverse weather ranging from cold in the north to very hot in the south. Lastly, all three types of HVAC appliances (ACs, COs, and MFs) have been deployed extensively throughout Iraq and a plethora of such appliances are offered in Iraqi markets.

The organization of the paper is as follows: Section II introduces the approach and its operation. Section III describes the implementation, the integrated development and emulation environment and the detail of a representative case study. Section IV provides an evaluation of the performance of the approach together with a discussion of results for three mitigation mechanisms. Section V contains conclusions. \#

\section{THE PROPOSED SYSTEM}

\section{A. System Structure}

The proposed system hardware comprises local controllers deployed in each dwelling of a residential area. Each controller is equipped with temperature and humidity sensing capabilities. Controllers are connected to a main controller located at the local substation with connections to all neighbouring generation facilities. Fig. 1 shows a typical layout of the system. A number of agents are defined to carry out key negotiation tasks and
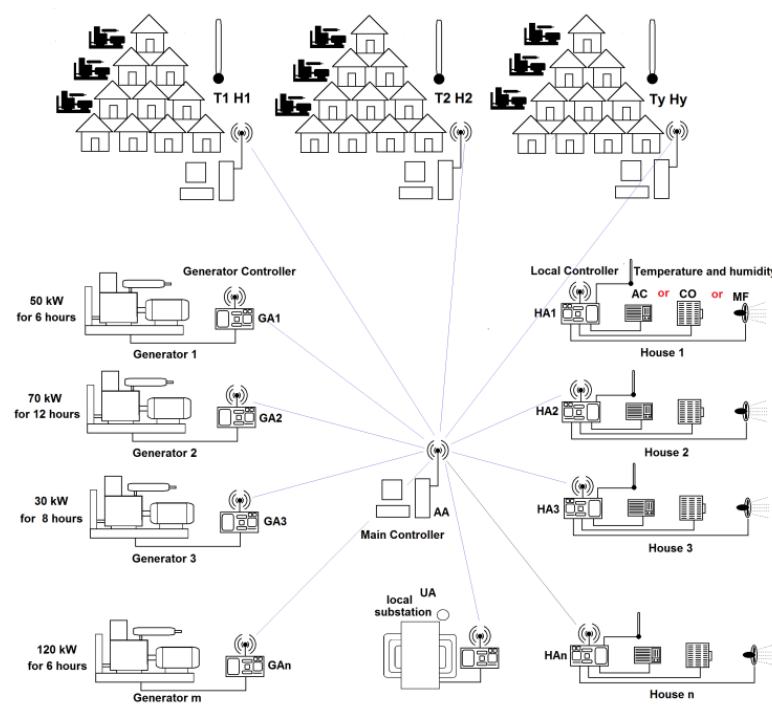

Fig.1 Typical system layout.

manage the re-distribution of power resources. Each dwelling is assigned a 'House Agent' (HA) responsible for the management 
of all appliances, including ON/OFF and proportional control of each appliance, sensing ambient temperature and humidity, and performing data exchange with other HAs and with the 'Administrative Agent' (AA). Houses are clustered and cluster heads are assigned.

The mix of generation facilities is managed through 'Generation Agents' (GAs), clustered with a cluster head. GAs map the amount of power available from a certain generator and at what time. A 'Utility Agent' (UA) is tasked with providing utility power delivery schedules. All agent activities are orchestrated by the AA. All main controllers in all sub-districts are connected together and temperature, humidity, available surplus power, and number of serviced customers are exchanged periodically. All AAs evaluate their share of power according to the exchanged information.

\section{B. System Operation}

The system aims to provide an adequate air conditioning level in addition to powering basic household appliances to consumers within wide geographies through swapping HVAC options with different demands (ACS, COs, and MFs). Each substation hosts the system's main controller that operates in a semi-central fashion and local controllers are placed at customers' houses. It is assumed that all main controllers in all substations are able to communicate with each other. Each main controller monitors the temperature, humidity, amount of surplus power in its area, and population to support and exchanges this data with all other main controllers. Based on these factors, each controller decides whether it is best to use ACs, COs, or MFs in its vicinity. All areas are powered from two sources: the intermittent limited utility that is periodically turned on and off and the available surplus power. In severe weather conditions, i.e. if temperature or humidity is too high, air cooling is not sufficient. The proposed system offers a practical solution for such highly distributed problem by offering such areas power to one AC per house for all dwellings until the weather relents.

The required power is provided by available surplus standby power and complemented by a continuous utility share so that the overall amount of power is sufficient to power the base and one AC load in all dwelling. In non-humid areas the remaining utility power is divided into ON/OFF periods with the OFF gaps powered by surplus power from local standby generation. At the local level (dwellings connected to a single substation) the system provisions power to basic household appliances and one HVAC appliance whose type depends on available power. All standby generation and appliances are connected to the LowVoltage distribution network. The system HVAC appliance selection pool contains three types viz. $\mathrm{AC}, \mathrm{CO}, \mathrm{MF}$, each differing in power consumption. At the outset the AA interrogates the generation cluster head to determine the available surplus power within its cluster. The generation cluster head reports the amounts, availability times, and periods of any surplus power stream. The AA aggregates all streams into one daily schedule of surplus power availability. Generators are ranked according to their ownership into: public, private, commercial and hospital. Public resources provide power free of charge as they are owned by local government and this free donation of surplus power matches their political need to alleviate cyclic blackout burden.

Private generation is costly to deploy and operate, thus it participates in the mitigation process as a fall back strategy.
Commercial generation resources are recruited and the local government has offered their owners support in the form of free fuel and half the cost of power they produce; the other half is paid by the consumer. Hospitals are powered continuously and their standby generation is used as a backup in case no other alternative is available. The donated surplus power amount changes from time to time; as the generation profiles change so does the donation patterns. All available surplus power is mapped into a single table referred to as the "power aggregation table' summarized and a 24 hour power profile is extracted.

During the utility OFF periods, the AA interrogates the power aggregation table securing a snapshot of the amount of available surplus power and its duration, calculates the basic household demand, calculates the amount of remaining power to power HVAC, and allocates the best mix of HVAC devices to cover the needs of each dwelling using optimization. The result is the provision of a fairly-allocated best-possible level of cooling for all dwellings. For example, in a time zone extending for 120 minutes and for 20 houses, if the optimization yields $(\mathrm{AC}=5$, $\mathrm{CO}=15, \mathrm{MFs}=0)$ the system then establishes a single HVAC cluster containing the selected HVAC appliances (5 ACs and 15 $\mathrm{COs})$. In this case 5 dwellings have the usage right to operate ACs, 15 houses COs, and there is thus no need for the use of MFs. If the number of ACs, COs, and MFs is not divisible, the system readjusts the number and mix of $\mathrm{ACs}$, COs, and MFs so that every dwelling is allocated a fair share of cooling. The number and type of HVAC appliances in the cluster is governed by the amount of available surplus power; thus a cluster is subject to dynamic changes in composition depending on the amount of surplus power. In essence the system also executes supply-demand matching. Each dwelling then is able to use its HVAC usage right until the end of interval, at which the surplus power is fixed. $\mathrm{AC}, \mathrm{CO}$, and $\mathrm{MF}$ usage rights are circulated among all dwellings in a fair way such that every dwelling will have the same amount of usage time for each HVAC appliance. The proposed system is semi-central in the sense that some activities are central under the supervision, control, and coordination of the AA while the rest are distributed, carried out through command-based packetized inter-agent negotiation.

Fig. 2 shows the functional block diagram of the proposed system. All generation streams are recorded as the local generation pool and their data are forwarded to the local generation dispatcher who produces a unified schedule and passes it to the system data pool which hosts it, in addition to size of served customers, ambient temperature and humidity. The last two parameters originate from the sub-district estimator which decides which type of HVAC appliances are to be activated. All this data are forwarded to the power source estimator which briefs the power source optimizer on the amount of available power, durations, ownership, kWh cost, temperature, humidity, and customer base. The optimizer evaluates the optimum utilitygeneration mix and distributes its decision to the power scheduler that re-distributes the selected power sources data to the power coverage module responsible for providing sufficient power to all base load appliances. After notification, the appliance dispatcher activates the right sockets of the targeted appliances. The appliance dispatcher also controls the HVACs appliances. The HVAC adaptive cluster is established depending on the available power stored within the power scheduler and passes it to the HVAC distribution selector responsible for selecting the correct HVAC which in turn is input to the cluster optimizer. After 
evaluating the optimum cluster, the appliance dispatcher activates the appropriate combination of HVAC appliances.

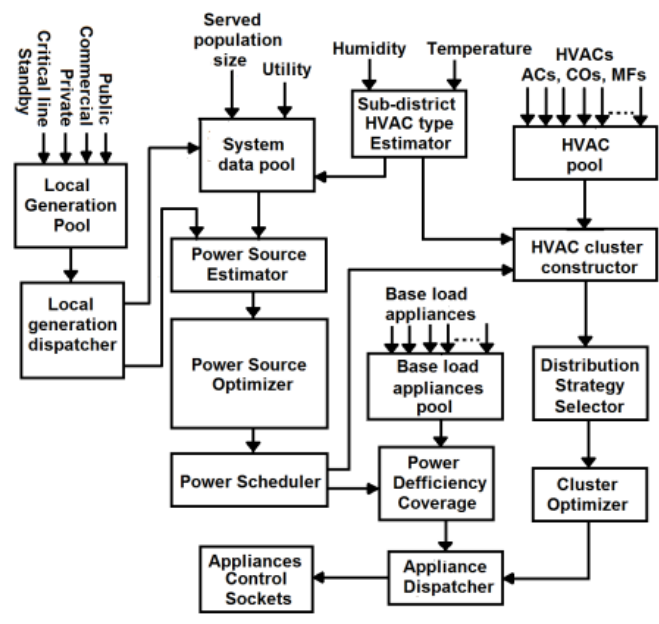

Fig.2 Proposed system functional block diagram.

\section{IMPLEMENTATION}

\section{A. Application}

The solution is applied to manage chronic cyclic blackouts in Basra, Iraq. Due to the long war (1980-2003), the bulk of the Iraqi generation, transmission, and distribution network was destroyed which precipitated severe power shortage and deep chronic cyclic blackouts. In parallel the extensive refurbishment of the Iraqi housing infrastructure (and thus deployment of appliances) has resulted in increasing the power demand. Basra is also subject to very hot weather; summer day temperatures can reach $56^{\circ} \mathrm{C}$ [19].

A detailed field survey was conducted to assess the problem, map available resources, and the factors affecting supply through a series of meetings with power administration personnel in Basra. A residential quarter -'Kafaat' - was selected as a suitable test environment. Kafaat is assumed to be a standalone subdistrict for simplicity. Data of other sub-districts and the communication between them are generated during the simulation.

The field study identified the availability of a mix of public, commercial and private standby generation surplus throughout Kafaat e.g. the largest being a $10 \mathrm{MW}$ standby generation facility. Detailed LV distribution network maps with other technical support data were secured to mark the boundaries, loads, transformers, house connections needed to setup the 20 house test environment.

As it was mentioned earlier and in an attempt to alleviate the power shortage burden, the local authorities in Basra have proposed a two part incentive scheme offered to all commercial and private standby generation facilities participating in providing power to residential areas. The local government will supply fuel to these facilities free of charge and will also bear the cost of half the generation fees for commercial users, the other half being charged to consumers.

\section{B. System simulation}

In order to verify the proposed solution, an Integrated Development Environment (IDE) was designed. IDE comprises demand generation based on house, family, and individual daily routine from data gathered from the field survey. IDE also generates residential demand profiles to test the performance of the proposed solution based on gathered data. IDE includes ON/OFF and proportional appliance control, demand analysis, house and family profiles, generation selection mechanisms, a graphics area, table display, message display and graphics control panel. It is assumed that the controller that hosts the HA is connected wirelessly to the central controller at the local substation which houses the AA. It is also assumed that all subdistrict controllers have some means of communication between them. All residential demand data are simulated for Kafaat only.

\section{RESULTS AND DISCUSSION}

The system mitigates the effects of cyclic blackouts for relatively wide area geographies divided into districts and subdistricts subject to diverse weather conditions. Since the solution partly relies on the use of EACs, the operation of which is highly dependent on the humidity level in the air, the solution monitors the targeted area humidity levels. Humid sub-districts are permitted to use ACs while the remainder use an optimized collection of ACs, COs, and MFs.

The first part of the results focuses on local system operation (within a single sub-district), showing how the system manages power shortage, covering the base load and HVAC demand. In the second part the dynamics of the wide area operation mode (all sub-districts) is addressed

\section{A. Local operation mode}

Fig. 3 shows a normal summer demand profile for 20 dwellings; no commercial, industrial, agricultural, religious, social, or sport areas are considered. Air conditioning is the dominating component in this demand and its footprint during night hours' is evident. Hour 8-14 are characterised by a low demand since the majority of people are at work or school.

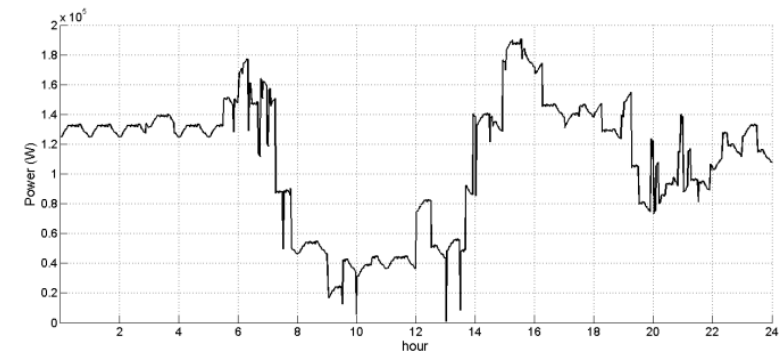

Fig.3 Summer demand profile for 20 residential dwellings.

Fig. 4 illustrates a simulation result showing the manner in which the system manages the demand of 30 houses. For illustration purposes and relevant to all scenarios under consideration, it is assumed that no utility supply is available and the base load is excluded in order to focus only on HVAC demand coverage. The 'blue' graph shows the collected surplus power while the 'red' one shows the amount of power required for HVAC appliances only. From these graphs it can be seen that within hour 1-6 there is sufficient power to operate $15 \mathrm{ACs}$ and $15 \mathrm{COs}$, so the time 
zone period is divided into two sub zones; within hour 7-8 no surplus power is available; within hour 9-12, a modest amount of power has been secured, sufficient to power $6 \mathrm{ACs}$ and $24 \mathrm{COs}$. Thus the time is divided into $(24+6) / 6=5$ sub-zones and since the time zone is 240 minutes long, then each sub-zone is $240 / 5=$ 48

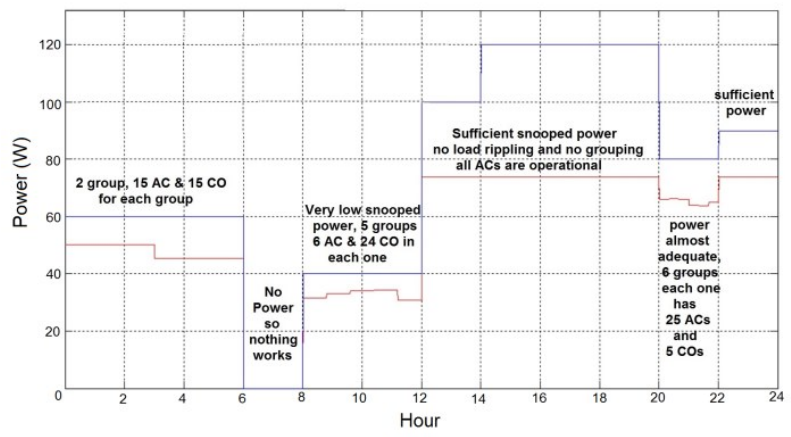

Fig.4 SnP and HVAC demand for 30 houses.

minutes long. Each house will have $1 \times 48$ minutes ACs usage time and 4x48 minutes $\mathrm{CO}$ usage time. Within hours 13-20 and 23-24 there is sufficient power to operate all ACs (one for each dwelling). Within hour 21-22, the power level is such that 25 $\mathrm{ACs}$ and 5 COs are active; the sub-zone period is $120 / 6=20$ minutes i.e. every dwelling has $5 \times 20$ minutes $\mathrm{AC}$ usage time and $1 \times 20$ CO usage time. It is worth noting that a certain level of surplus power under-utilisation occurs.

Fig. 5 shows the summer demand for 20 dwellings under $4 \times 2$ cyclic blackouts ('black') and the bridging of the no-power gaps in the demand by the proposed solution ('blue') consisting of two components, the base load and HVAC demands. The latter is also plotted ('red'). Both demand components are powered from surplus power ('purple').

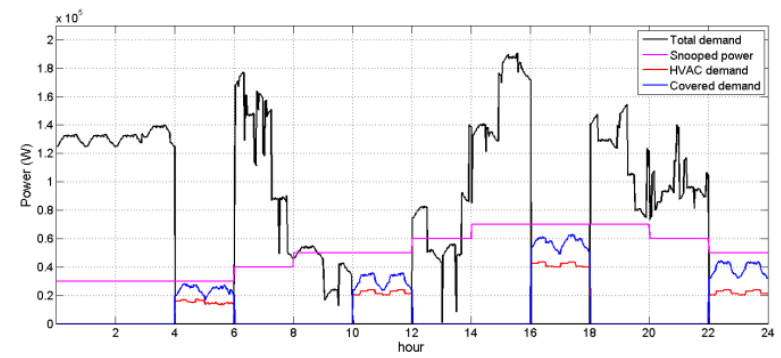

Fig. 5 Base load, surplus power, total and HVAC demands.

The overall demand is shown in Fig.6. During cyclic blackout periods, the proposed solution covers the base load demand from surplus power and also uses the surplus to power HVAC appliances after optimization. There are no limits or constraints on the demand during utility on hours, so customers can sustain their normal power usage patterns. The amount of unused surplus power varies depending on base load demand and the optimization and equal distribution of HVAC appliances. The surplus power during utility on hours is not used. As is evident, the system has sustained base load and HVAC demand efficiently and has smoothed the demand profile. In all relevant figures, payback is not considered because it was found that in hot countries customers tend to leave their ACs' thermostat set the maximum [20].

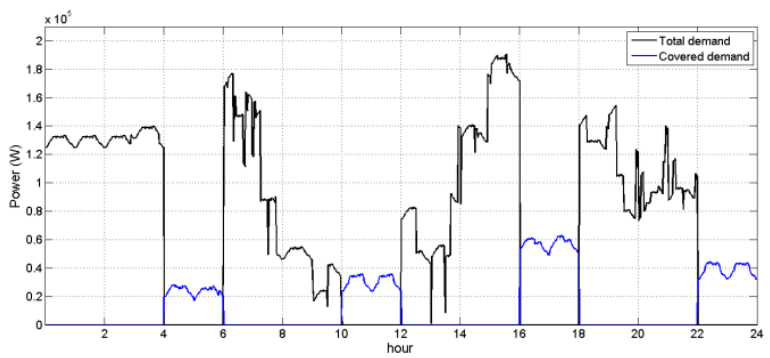

Fig.6 The mixed demand profile.

\section{B. Wide area operation mode}

During high humidity or severe temperature periods the system offers AC usage rights to all houses within the area. To power these houses, the system uses the limited utility. Thus both base and one AC loads are powered continuously, i.e. converting the ON/OFF into a continuous supply for a limited period one shown in Figures 9-11. Fig. 7 shows the normal HVAC demand of 20 houses (blue) and the continuously-fed limited single AC/house HVAC load (red).

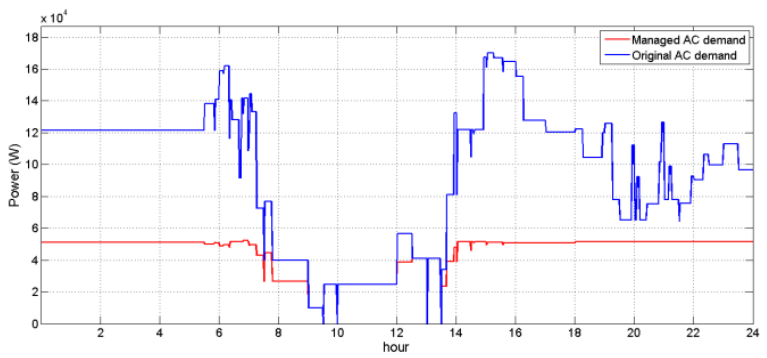

Fig.7 HVAC demand under extreme weather conditions.

The reduction in overall HVAC demand is evident, the system achieving nearly a $65 \%$ reduction. The other noteworthy feature is that the resulting single AC/house demand is nearly flat. Fig. 8 shows the components of this daily demand (without cyclic blackout) which must be covered by the utility-surplus power mixture.

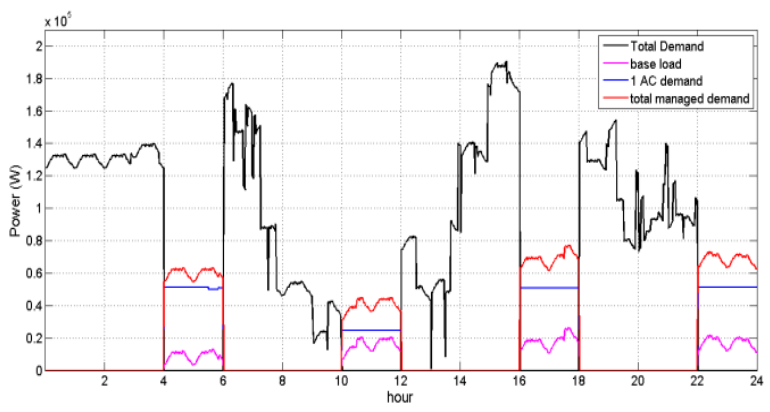

Fig.8 Demand composition under extreme weather. 
Fig. 9 shows the original demand of the 20 houses and their demand after management by the system. The resulting demand is nearly one third of original one following a near flat profile.

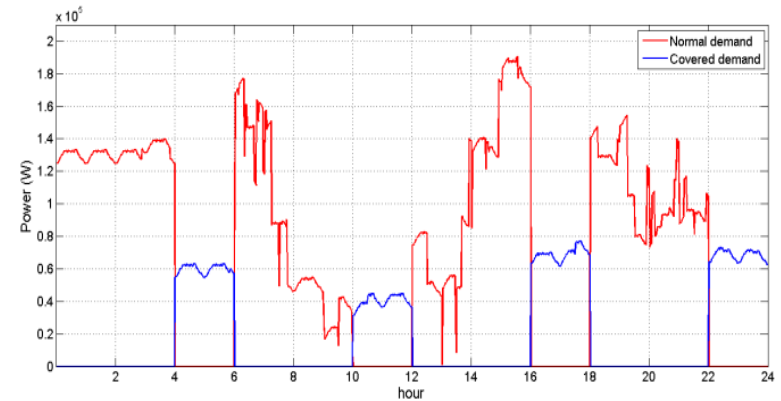

Fig.9 Original and managed demand.

\section{CONCLUSIONS}

A multi-agent distributed DSM-based system with a supplydemand matching capability has been used to sustain residential base load and HVAC demand during cyclic blackouts in wide geographies having diverse weather conditions. The systems power different HVAC appliance according to the weather conditions at that area. Hot and/or humid areas get ACs only, while the rest get a combination of air conditioners, air coolers, and/or mist fans which are clustered in a dynamically updated pool of cooling resource used to service customers on a oneHVAC appliance per dwelling basis. The mix and number of air conditioners, air coolers, and mist fans depends on the amount of available power. HVAC usage rights are distributed fairly amongst all customers. To power these appliances during a cyclic blackout, the system interrogates all nearby generation for power surplus, aggregates all excess power and deploys it judiciously to provision power to better meet demand. The system clusters dwellings and assigns cluster heads to each, and recruits different types of agents for the negotiation of resources. Results show that the approach is successful in sustaining the power to basic household and one HVAC appliance per dwelling. The resulting demand profile also is also smoothed. In summary, to mitigate cyclic blackouts in wide areas and manage extreme weather conditions, the use of COs and MFs in tandem with ACs is a viable option that meets user demands.

\section{REFERENCES}

[1] G. o. I. Ministry of power, "Grid Disturbance in Northern Region on July 30, 2012 and in Northern, Eastern \& North-Eastern Region on July 31 2012," Ministry of power, Government of India, New Delhi, India, 2012.

[2] M. Vaiman , P. Hines, S. Norris , A. Pitto , Y. Wang, G. Zweigle, J. Jiang and M. Papic, "Mitigation and Prevention of Cascading Outages: Methodologies and Practical Applications," in IEEE Power and Energy Society General Meeting (PES), Vancouver, Canada, 2013.

[3] D. E. Newman, B. A. Carreras, V. E. Lynch and I. Dobson, "Exploring Complex Systems Aspects of Blackout Risk and Mitigation," IEEE TRANSACTIONS ON RELIABILITY, vol. 60, no. 1, pp. 134-143, 2011.

[4] K. L. J. Z. P. L. C.-C. Yamashita, "Analysis and Control of Major Blackout Events," in IEEE/PES Power Systems Conference and Exposition PSCE '09. , Seattle, USA, 2009.

[5] Z. Shaobo and S. Zhanhui, "Challenges and opportunities in emergency management of electric power system blackout," in International Conference on E-Product E-Service and E-Entertainment (ICEEE), Henan, 2010.

[6] B. Enacheanu, M. Fontela, C. Andrieu, H. Pham, A. Martin and Y. Besanger, "New control strategies to to prevent blackout: Intentional islanding operation in distributed networks," in 18th International Conference on Electricity Distribution, Turin, 2005.

[7] S. Rahman, M. Pipattanasomporn and Y. Teklu, "Intelligent Distributed Autonomous Power Systems (IDAPS)," in IEEE Power Engineering Society General Meeting, Tampa, USA, 2007.

[8] S. S. Ahmed, N. C. Sarker, z. B. Khairuddin, M. R. B. Abd Ghani and H. Ahmad, "A Scheme for Controlled Islanding to Prevent Subsequent Blackout," IEEE TRANSACTIONS ON POWER SYSTEMS, vol. 18, no. 1, pp. 136-143, 2003.

[9] M. F. Baba, "smart grid with ADSL connection for solving peak blackout in west bank," in First International Conference on Renewable Energies and Vehicular Technology (REVET), Hammamet, Tunsia, 2012.

[10] H. Seyedi and M. Sanaye-Pasand, "New centralised adaptive load-shedding algorithms to mitigate power system blackouts," IET Generation, Trans. \& Distribution, vol. 3, no. 1, p. 99-114, 2008.

[11] M. K. Kouluri and R. K. Pandey, "Intelligent Agent Based Micro grid Control," in 2nd International Conference on Intelligent Agent and MultiAgent Systems (IAMA), Chennai, 2011.

[12] S. Bashash and F. K. Hosam, "Modeling and control insights into demandside energy managment through setpoint control of thermostatic loads," in American control conference, San Francisco, USA, 2011.

[13] R. Lokapure and J. Joshi, "Energy conservation through roof surface evaporative cooling for air conditioning system," International Journal of Scientific and Research Publications, vol. 2, no. 6, pp. 1-5, 2012.

[14] J. K. Kok, C. J. Warmer and I. G. Kamphuis, "PowerMatcher: Multiagent Control in the Electricity infrastructure," in Proceedings of the fourth international joint conference on Autonomous agents and multiagent systems (AAMAS '05), New York, USA, 2005.

[15] M. Osborn, "Experiences in Aggregating Distributed Generation For System Benefit," in IEEE Power Engineering Society General Meeting, Denver, USA, 2004.

[16] A. Mansoor, S. A. Mansoor and S. A. K. Masud, "Aggregation and Dispatching of Captive Generator Sets for System Load Reduction and Capacity Relief," in Second International Conference on Electrical and Computer Engineering, Dhaka, Bangladesh, 2002.

[17] M. Ako, R. Hara, H. Kita and T. Funabashi, "A Method for Balancing the Supply and Demand in an Isolated System Consisting of Voltage Control Type Inverters in FRIENDS," IEEE Trans. on Power Systems, vol. 25, no. 2, pp. 1098 - 1105, 2010.

[18] F. Saffre, H. Hildmann and S. Nicolas, "Modeling Quality of Service vs. Peak Reduction trade-offs in A/C-based Demand-Side Management," in IEEE First International Workshop on Smart Grid Modeling and Simulation (SGMS), Brussels, 2011.

[19] WeatherSpark, "Basra International Airport Weather station," 1242014. [Online]. Available: http://weatherspark.com/\#!graphs;ws $=32872$.

[20] L. a. A. T. C. a. L. Proctor Engineering Group, "High EER at $46^{\circ} \mathrm{C}$ Kingdom of Saudi Arabia Air Conditioner Project," Saudi Ministry of Water and Electricity, Riyadh, Saudi Arabia, 2013 